\title{
Trasplante alogénico de progenitores hematopoyéticos para síndromes de falla medular, experiencia de la Unidad de Trasplante de Médula Ósea de la Clínica de Marly
}

\author{
Allogenic stem cell transplantation for bone marrow failure syndrome, \\ experience from the Bone Marrow Transplant Unit at the Clínica de Marly
}

\author{
Virginia Abello',2, Licet Villamizar1,2, Enrique Pedraza1', Herman Esguerra', Carmen Rosales', Adriana Linares' \\ Manuel Rosales', Javier Figueroa', William Mantilla', Claudia Casas',2, Nathalia Villaroya' \\ 'Unidad de Trasplante de Médula Ósea, Clínica de Marly (Bogotá, Colombia). \\ 2 Fundación Universitaria de Ciencias de la Salud, Hospital de San José (Bogotá, Colombia).
}

\section{Resumen}

Introducción: El trasplante alogénico de precursores hematopoyéticos (TAPH) es considerado como el tratamiento de elección para los pacientes menores de 40 años con síndromes de falla medular (SFM), así como para los pacientes mayores quienes han fallado al tratamiento inmunosupresor.

Métodos: Se realizó un estudio retrospectivo de los pacientes pediátricos y adultos con diagnóstico de SFM sometidos a trasplante alogénico en una única institución de Bogotá (Colombia). El desenlace primario fue la supervivencia global (SG) a cinco años mediante el método de Kaplan-Meier. Se utilizó la prueba log-rank para determinar el efecto de varios factores sobre la SG. Se exploró la estancia hospitalaria, la presentación de enfermedad injerto contra huésped aguda (EICHa) grados II-IV y la falla secundaria del injerto.

Resultados: Entre enero de 1993 y agosto de 2011 se realizaron 74 trasplantes en 70 pacientes. En 70 casos los donantes fueron hermanos HLA idénticos, en 3 se obtuvo de la sangre de cordón umbilical y en 1 fue a partir de un hermano haploidéntico. Los condicionamientos más utilizados fueron: Cy-Flu-ATG no mieloablativo (22), Cy-ATG (33) y Cy (12). Cuatro pacientes recibieron condicionamientos con alemtuzumab. Antes del 2004, la mayoría de los pacientes recibió profilaxis para EICH con Cs y MTX; después del 2004, se utilizó Cs y MMF. Dos pacientes murieron el día de la infusión de progenitores hematopoyéticos. Para el primer TAPH, el injerto de neutrófilos para los 68 pacientes se documentó en promedio a los 11.5 días, de manera similar entre los esquemas de acondicionamiento. Diez pacientes (14\%) presentaron una falla secundaria del injerto, de quienes 5 murieron en promedio a los 178 días después del TAPH. De los 5 restantes, 2 se encuentran con dependencia transfusional y 3 fueron trasplantados exitosamente por segunda vez. La EICHa G II-IV se presentó en el 30\%, del cual el $46.7 \%$ murió por complicaciones relacionadas; esta complicación fue más frecuente en los pacientes cuyos regímenes no incorporaban ATG. Para una mediana de seguimiento de 18.4 meses (rango 4.3-45.3), la SG fue del $69.1 \%$ y varió de acuerdo con el régimen de acondicionamiento $(p=0.0008)$. Con el condicionamiento no mieloablativo, se lograron unas estancias hospitalarias más cortas, los pacientes presentaron menos días de fiebre, se requirieron menos transfusiones y menos días de nutrición parenteral. No hubo diferencias significativas relacionadas con la edad $(p=0.67)$. Se observó una SG mayor pero no significativa en los pacientes tratados después del año 2004 ( $p=0.38$ ).

Conclusiones: La presente experiencia demostró que el TAPH es una opción terapéutica primordial que ofrece una mejor SG para los pacientes con SFM, independientemente de la edad y el año de la realización, y sugiere una ventaja del condicionamiento no mieloablativo basado en fludarabina.

Palabras clave: anemia aplásica, anemia de Fanconi, aplasia pura de células rojas, trasplante de células madre hematopoyéticas, acondicionamiento pretrasplante. 


\begin{abstract}
Background: Allogeneic hematopoietic stem cell transplantation (allo-SCT) is considered as the treatment of choice for newly diagnosed patients with bone marrow failure syndromes (BMFS) younger than 40 years, and for older patients that have failed immunosuppressive therapy.

Methods: This is a retrospective study of BMFS pediatric and adult patients receiving allo-SCT at a single institution from Bogota, Colombia. The primary outcome was five-year overall survival (OS), determined by the Kaplan-Meier method. Log-rank test was used to determine the impact of various factors over OS. In-hospital stay, grade II-IV acute graft versus host disease (aGVHD) and secondary graft failure were explored.

Results: Between January 1993 and August 2011, 74 transplants were performed in 70 patients. 70 grafts were obtained from HLA identical siblings, 3 from unrelated umbilical cord blood and 1 from a haploidentical sibling, were obtained from HLA identical siblings, 3 from unrelated CBU and 1 from a haploidentical sibling. Conditioning regimens used were: RIC Cy-Flu-ATG (22), Cy-ATG (33) and Cy (12). 4 patients were conditioned with alemtuzumab containing regimens and 3 with other. Before 2004 most patients received Cs and MTX as GVHD prophylaxis, after 2004 Cs and MMF was used in the majority. Two patients died during the progenitors infusion. Engraftment for 68 evaluable patients at first allo-SCT ocurred at an average of 11.5 days, similarly between conditioning regimens. 10 patients (14\%) had secondary graft failure of whom five died at a mean of 178 days after the allo-SCT. Five patients remain alive, two are transfusion-dependent and three succeeded a second allo-SCT. aGVHD occurred in 30\% of patients of whom $46.7 \%$ died due to related complications; aGVHD was more frequent with ATG non-containing regimens. For a median follow-up of 18.4 months (range: 4.3- 45.3) OS was $69.1 \%$ and varied according to conditioning combination $(p=0.0008)$. There was no significant difference for OS related to age $(p=0.67)$. A greater but not significant OS was observed for allo-SCT performed after than before $2004(p=0.38)$. Conclusions: This experience showed that allo-SCT is a fundamental therapeutic option for patients with BMFS treated with allo-SCT, offering a better OS regardless the age and year of performance, suggesting an advantage for RIC over other conditioning regimens.
\end{abstract}

Key words: aplastic anemia, Fanconi anemia, pure red-cell aplasia, hematopoietic stem cell transplantation, transplantation conditioning.

\section{Introducción}

Los síndromes de falla medular (SFM) son un grupo de enfermedades que comprenden desde los trastornos congénitos de la hematopoyesis, como la anemia de Fanconi, la aplasia pura de la serie roja (APSR) y el síndrome de Blackfan-Diamond, hasta aquellos adquiridos, como la aplasia severa de médula ósea (ASMO); este último síndrome constituye el tipo más frecuente de falla medular en los adultos, y su fisiopatología se considera predominantemente autoinmune'.

El trasplante alogénico de progenitores hematopoyéticos (TAPH) es el tratamiento de elección en pacientes jóvenes con diagnóstico de ASMO y otros síndromes de falla medular, que tienen un donante HLA idéntico independientemente de su disponibilidad familiar ${ }^{2-5}$ esta estrategia ha demostrado supervivencias libres de enfermedad reportadas entre el $60 \%$ y $95 \%$ en varios estudios $^{6-11}$. En los pacientes mayores de 40 años, se ha considerado clásicamente que el TAPH está indicado después de la falla de la terapia inmunosupresora4; sin embargo, con el soporte actual, algunos reportes sugieren resultados superiores a la inmunosupresión. Específicamente, con el uso de fludarabina como parte del acondicionamiento, se han reportado tasas de supervivencia de hasta un $77 \%$ a cinco años ${ }^{12,13}$.
En este estudio, se pretende realizar una descripción retrospectiva de los pacientes con SFM sometidos a trasplante alogénico durante los últimos 18 años en la Unidad de Trasplante de Médula Ósea (UTMO) de la Clínica de Marly.

\section{Materiales y métodos}

Se diseñó un estudio retrospectivo, cuyo objetivo principal consistió en describir la supervivencia a cinco años de los pacientes pediátricos y adultos sometidos a TAPH en una única institución (considerada como centro de remisión a nivel nacional), en el período comprendido entre enero de 1993 y agosto del 2011. Para el diagnóstico de ASMO, se tuvieron en cuenta los criterios diagnósticos de la British Society of Hematology con sus respectivos grados ${ }^{2}$.

La estadística descriptiva se presentó mediante medidas de tendencia central y de dispersión para las variables cuantitativas, y en porcentajes para variables cualitativas. Se describió la incidencia acumulativa de enfermedad injerto contra huésped aguda (EICHa) grado II a IV definida según los criterios de Glucksberg ${ }^{14}$, así como la falla del injerto y la mortalidad. La supervivencia global (SG) se determinó utilizando el método de Kaplan-Meier y sus factores relacionados (la edad, 
el tipo de quimioterapia de condicionamiento, el año de tratamiento, el número de transfusiones y el tiempo del diagnóstico al trasplante en la supervivencia) fueron analizados empleando la prueba de log-rank. Todos los análisis estadísticos fueron realizados en el programa estadístico Stata v 10.0.

Para el diagnóstico de los eventos objetivo del estudio se tomaron en cuenta las siguientes definiciones:

Falla primaria del injerto: se definió como la falta de recuperación hematológica (neutrófilos $>500$ y plaquetas $>20.000$ por más de 3 días consecutivos) en pacientes que han sobrevivido más de 21 días después del $\mathrm{TAPH}^{15}$.

Falla secundaria del injerto: se definió como la recuperación de la hematopoyesis completa (recuperación del recuento de neutrófilos y plaquetas) o parcial (recuperación del recuento de neutrófilos sin recuperación de plaquetas), seguida de pancitopenia, con una médula ósea severamente hipocelularin.

Supervivencia: se calculó a partir del día del trasplante hasta el día de la muerte, excluyendo los pacientes que fallecieron el día del trasplante, y se expresó en meses.

Politransfusión: Se definió como presente en aquellos pacientes que habían sido sometidos a más de 10 episodios transfusionales desde el diagnóstico hasta el día del TAPH.

\section{Resultados}

Desde enero de 1993 hasta agosto del 2011, se realizaron 74 trasplantes en 70 pacientes. La mayoría de los pacientes tenían diagnóstico de ASMO (74.3\%, n = 52). Se incluyeron en el análisis dos pacientes con APSR, debido a que esta entidad presenta una historia natural e implicaciones terapéuticas similares a la ASMO.

En la tabla 1 se describen las características clínicas de los pacientes trasplantados. El tiempo promedio desde el diagnóstico hasta el TAPH para el grupo fue de 26.5 meses (2.1-143) y la mayoría de los donantes fueron hermanos HLA idénticos. Los progenitores hematopoyéticos (PH) se obtuvieron en un $86.4 \%$ ( $n=64)$ de sangre periférica recolectados mediante aféresis después de movilización con factores estimulantes de colonias. Se utilizaron PH de médula ósea en un porcentaje pequeño de pacientes $(n=6,8.1 \%)$. Tres TAPH se realizaron con progenitores hematopoyéticos de sangre de cordón umbilical (SCU), todos ellos en
Tabla 1. Características clínicas y demográficas de los pacientes trasplantados $(\mathrm{n}=70)$

\begin{tabular}{|l|c|}
\hline \multicolumn{1}{|c|}{ Característica } & $\mathbf{n}(\%)$ \\
\hline Género, F/M & $28(40) / 42(60)$ \\
\hline Edad (años)* & $25.4(4-60)$ \\
\hline Procedencia: Bogotá/Fuera de Bogotá & $40(57.2) / 30(42.8)$ \\
\hline Tiempo de evolución (meses)* & $26.5(2.1-143)$ \\
\hline Politransfundido & $43(61.1)$ \\
\hline ATG/LG previa & $14(20)$ \\
\hline CMV positivo & $49(70)$ \\
\hline Diagnóstico & \\
\hline Aplasia adquirida & $52(74.3)$ \\
\hline Anemia de Fanconi & $16(22.9)$ \\
\hline Aplasia pura serie roja & $2(2.9)$ \\
\hline
\end{tabular}

Promedio (rango).

pacientes con anemia de Fanconi. En un caso se usaron $\mathrm{PH}$ obtenidos de la sangre periférica de un donante haploidéntico, para rescatar a uno de ellos que presentó falla primaria del injerto (tabla 2).

Hasta el año 2006, el protocolo de condicionamiento más utilizado fue la combinación de ciclofosfamida (Cy) a dosis de $50 \mathrm{mg} / \mathrm{kg} /$ día por 4 días y globulina antitimocítica (ATG) a dosis de $3 \mathrm{mg} / \mathrm{kg} /$ día por 4 días (Cy-ATG). Entre los años 1998 y 2005, 12 pacientes fueron condicionados con un protocolo basado solo en Cy sin ATG. Este protocolo fue abandonado debido a los resultados desalentadores dados por una alta tasa de EICHa. De igual forma, durante este período, la profilaxis de EICHa estuvo compuesta de ciclosporina (Cs) a dosis de entre 3 y $5 \mathrm{mg} / \mathrm{kg} / \mathrm{día}$ desde el día -1, junto con metotrexate (MTX) a dosis de $20 \mathrm{mg} / \mathrm{m}^{2} / \mathrm{día}$ los días $+1,+3,+6$ y +11 .

A partir del año 2006, se realizaron cambios significativos en los protocolos de manejo de la UTMO implementando como acondicionamiento para los pacientes con ASMO un protocolo no mieloablativo denominado Cy-Flu-ATG, compuesto por Cy a dosis de 10 mg/kg/día,

Tabla 2. Características del trasplante $(n=74)$

\begin{tabular}{|l|c|}
\hline \multicolumn{1}{|c|}{ Característica } & n (\%) \\
\hline Fuentes de progenitores hematopoyéticos & $64(86.4)$ \\
\hline Sangre periférica & $6(8.1)$ \\
\hline Médula ósea & $3(4)$ \\
\hline Cordón umbilical & $1(1.4)$ \\
\hline Haploidéntico & \\
\hline Quimioterapia de condicionamiento & $33(44.6)$ \\
\hline Cy-ATG & $22(29.8)$ \\
\hline Cy-Flu-ATG no mieloablativo & $12(16.2)$ \\
\hline Cy & $4(5.4)$ \\
\hline Campath-Cy & $1(1.3)$ \\
\hline Campath-Cy-Flu & $1(1.3)$ \\
\hline Cy-Flu & $1(1.3)$ \\
\hline No anotado & Abreviaciones: Cy: ciclofosfamida, Flu: fludarabina, ATG: globulina antitimocítica, Campath: alemtuzumab.
\end{tabular}


fludarabina (Flu) a dosis de $30 \mathrm{mg} / \mathrm{m}^{2} /$ día y ATG a dosis de $2 \mathrm{mg} / \mathrm{kg} / \mathrm{día}$, por 4 días. También se modificó el protocolo de profilaxis de EICHa por Cy a dosis de entre $3 \mathrm{mg}$ y $5 \mathrm{mg} / \mathrm{kg} / \mathrm{día}$ desde el día -1, junto con micofenolatomofetil (MMF), por 28 días. Adicionalmente, se instituyó el uso de profilaxis antimicrobiana de rutina con levofloxacina de manera sistemática para todos los pacientes trasplantados (tabla 2).

La experiencia utilizando el esquema Cy-Flu-ATG demostró que el protocolo fue significativamente mejor tolerado, el cual resultó en un menor tiempo de hospitalización en comparación con Cy-ATG y Cy (promedio de 22.9, 32, 33 días, respectivamente), menos días de fiebre (promedio de 1.7, 6.7 y 7 días, respectivamente), menos días de alimentación parenteral por paciente (promedio de 0.1, 9, 4 días, respectivamente) y menos transfusiones de glóbulos rojos (promedio de 2.4, 3.8, 3.8 episodios transfusionales, respectivamente) y plaquetas (promedio de 4.5, 7.5, 9.7 episodios transfusionales, respectivamente). La información comparativa sobre los resultados obtenidos con los esquemas de acondicionamiento se ilustra en la tabla 3.

Dos pacientes, severamente politransfundidas, murieron por sepsis el día de la infusión de los $\mathrm{PH}$, por este motivo tales pacientes no fueron tenidos en cuenta para el análisis de la supervivencia. Una paciente murió por sepsis ocho días después del trasplante, por lo cual no puede ser tenida en cuenta para el análisis del injerto. Los resultados obtenidos durante el TAPH para los 68 pacientes evaluables se muestran en la tabla 3 . El injerto de neutrófilos para el primer trasplante se documentó en 66 pacientes de los 67 evaluables, en promedio a los 11.5 días, sin diferencias de acuerdo con los diferentes condicionamientos (tabla 3).

Tabla 3. Resultados clínicos de los tres tipos de condicionamiento más frecuentes en los pacientes trasplantados

\begin{tabular}{|c|c|c|c|}
\hline Resultado & $\underset{(n=12)}{C y}$ & $\underset{(n=33)}{\text { Cy-ATG }}$ & $\begin{array}{c}\text { Flu-Cy-ATG } \\
(n=22)\end{array}$ \\
\hline Edad & $28.7(6-57)$ & $21.1(4-57)$ & $29.9(4-60)$ \\
\hline Tiempo evolución - meses & $21.9(2.3-75.1)$ & $30(2.1-143)$ & $20.9(3.7-120.3)$ \\
\hline Células MNC (108/kg) & $5.06(1.2-109)$ & $14.7(3.4-54.7)$ & $16(0.11-59.1)$ \\
\hline Días de hospitalización & $33(24-36)$ & $32(20-94)$ & $26(17-62)$ \\
\hline Días aplasia neutrófilos & 16.6 & 15.9 & 15.8 \\
\hline Injerto neutrófilos & $13.2(9-16)^{*}$ & $11.8(7-21)^{* *}$ & $10.5(6-16)^{* * *}$ \\
\hline Días de fiebre & $7(1-22)$ & $6.7(0-20)$ & $2.4(0-15)$ \\
\hline Días HAP & $4(0-31)$ & $9(0-69)$ & $0.1(0-2)$ \\
\hline Transfusiones GRE & $3.8(2-8)$ & $3.8(0-21)$ & $2.1(0-9)$ \\
\hline Transfusiones plaquetas & $9.7(3-25)$ & $7.5(0-35)$ & $5(0-13)$ \\
\hline
\end{tabular}

Un niño de cinco años tuvo falla primaria del injerto después de un trasplante de sangre de cordón umbilical; la celularidad y la compatibilidad de la unidad transfundida eran adecuadas según los criterios de selección internacionales. Como rescate, se realizó un trasplante haploidéntico, utilizando progenitores hematopoyéticos de un donante intrafamiliar, con lo que el paciente logró un injerto adecuado, y permanece vivo a la fecha de este análisis, 22 meses después del TAPH, con signos clínicos de EICH crónica (EICHc) en la piel.

En 10 pacientes se evidenció una falla secundaria del injerto; 4 de ellos por abandono del tratamiento inmunosupresor; 2 de los 4 pacientes condicionados con alemtuzumab (anticuerpo monoclonal anti-CD52) y 4 distribuidos en los otros condicionamientos. De estos 10 pacientes, 5 murieron en promedio a los 178 días después del trasplante (rango 74-365), 3 recibieron un segundo trasplante de progenitores hematopoyéticos de sangre periférica exitosamente y están vivos sin presentar EICH; y 2 sobreviven a la fecha con dependencia transfusional.

La frecuencia de la EICHa grados II-IV fue del 30\% $(n=21)$, implicando una mortalidad secundaria directa o indirecta en 10 casos (47.6\%). Los pacientes restantes fueron manejados mediante el tratamiento específico, logrando el control de la patología. La EICHa fue más frecuente en el grupo condicionado con Cy, en comparación con los grupos condicionados con Cy-ATG y Cy-Flu-ATG (42\%, 30\% y 29\%, respectivamente). Ninguno de los pacientes que recibió acondicionamiento con alemtuzumab presentó EICHa.

Para una mediana de seguimiento de 18,4 meses, la SG en todo el grupo fue del $69.1 \%$ y se expone en la figura 1. Veintiuno de los 68 pacientes tenidos en cuenta en el análisis murieron. La principal causa de muerte fue la EICHa en los 10 casos descritos: la mortalidad según régimen por EICH se presentó en 5, 4 y 1 pacientes acondicionados con Cy, Cy-ATG y Cy-Flu-ATG, respectivamente. Las causas restantes de mortalidad fueron la sepsis (5 pacientes) y falla secundaria del injerto (5 pacientes, 2 de ellos por abandono del tratamiento inmunosupresor después del trasplante).

Dos factores se relacionaron significativamente con la SG. En primera instancia, la quimioterapia de acondicionamiento; al momento del análisis, el 94\% de los pacientes acondicionados con Cy-Flu-ATG se 


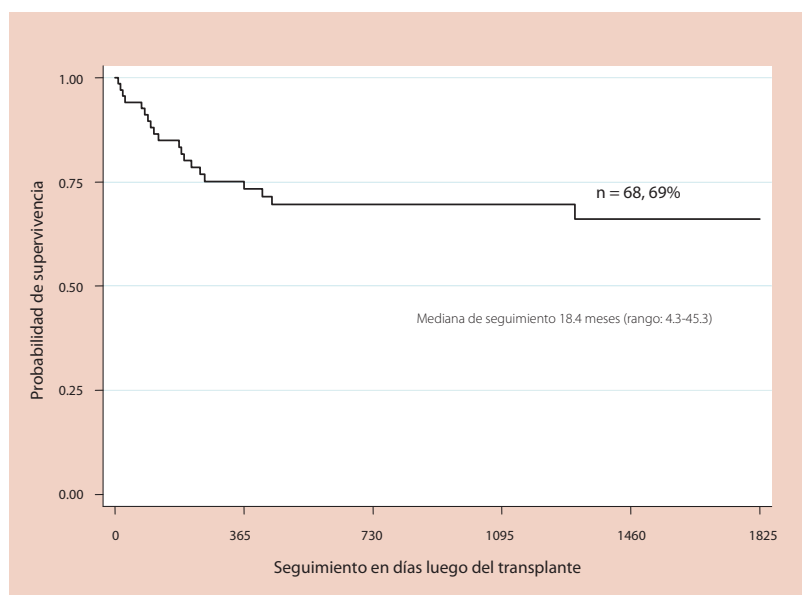

Figura 1. Supervivencia global a cinco años en pacientes con aplasia medular.

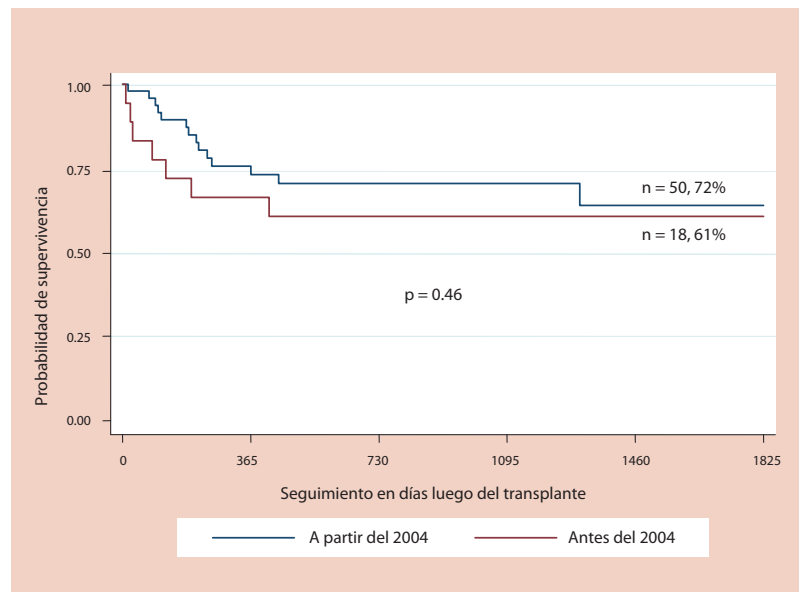

Figura 3. Supervivencia global a cinco años en pacientes con aplasia medular según tiempo de tratamiento.

encontraban vivos frente al 75 y $30 \%$ para los pacientes acondicionados con Cy-ATG y Cy, respectivamente $(p=0.0008)$ (figura 2). Adicionalmente, la fecha de realización del TAPH también tuvo una tendencia sobre la SG. La supervivencia para los pacientes trasplantados después del 2004 fue del 74\% frente al 61\% trasplantado antes de esta fecha ( $p=0.38$ ) (figura 3 ).

Por otra parte, no hubo diferencia significativa con respecto a la edad -menos de 40 años $73.3 \%(n=53)$ versus mayores de $4069.9 \%(n=15), p=0.67$ ) (figura 4). De todos los pacientes, 15 (22\%) eran mayores de 40 años; la supervivencia para ellos fue del $73.3 \%$ (murieron 4 de 15: 1 de los 4 condicionados con $\mathrm{Cy}$, 2 de los 3 condicionados con Cy-ATG y 1 de los 8 condicionados con Cy-Flu-ATG). Tampoco hubo diferencias de la SG según el número de transfusiones previas ni el tiempo entre el diagnóstico y el trasplante. Por el bajo

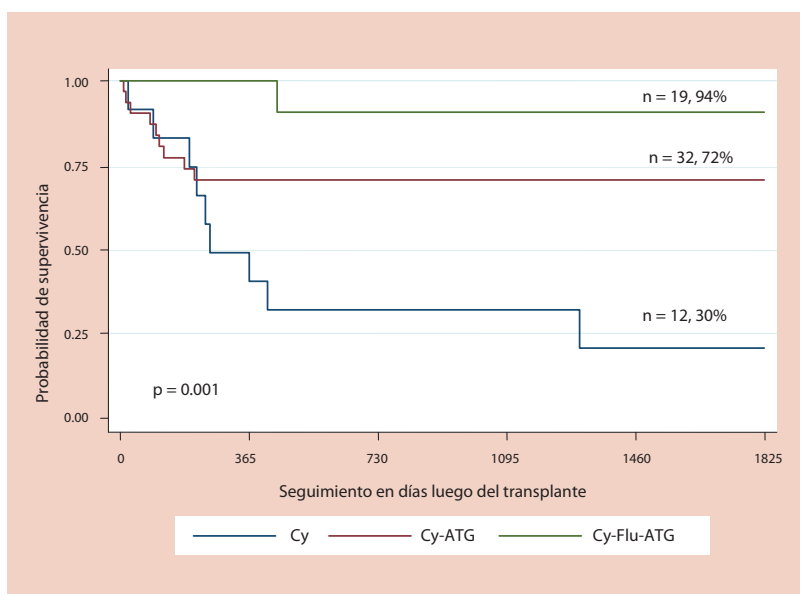

Figura 2. Supervivencia global a cinco años en pacientes con aplasia medular según quimioterapia.

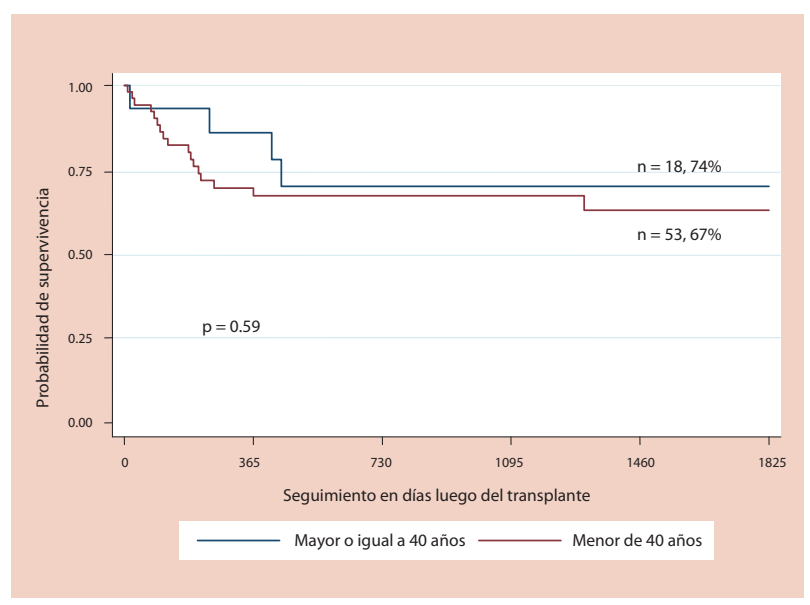

Figura 4. Supervivencia global a cinco años en pacientes con aplasia medular por grupos de edad.

número de trasplantes realizados con fuentes de $\mathrm{PH}$ diferentes a la sangre periférica, fue imposible definir alguna relación de este factor y la SG.

\section{Discusión}

Después de realizado el diagnóstico de ASMO mediante el establecimiento de celularidad de la médula ósea y el nivel de compromiso de los recuentos celulares ${ }^{16}$, la decisión de la terapia de primera línea depende de factores tales como la edad, la disponibilidad de un donante intrafamiliar HLA compatible y la severidad de la enfermedad ${ }^{4}$.

En los pacientes menores de 40 años, el TAPH ofrece una oportunidad de curación superior al 80\% a largo plazo, lo cual ha resultado en un drástico impacto desde la adición de la ATG a los protocolos 
de acondicionamiento9 . Lo anterior justifica su consideración como el tratamiento de elección de primera línea para esta población². Actualmente, existe una controversia entre los grupos de trabajo en TAPH para SFM en cuanto a la consideración sobre el límite superior de edad a partir del cual no se debería ofrecer la TAPH como terapia de primera línea, ya que, con los protocolos de condicionamiento clásicos, se observaron mayores tasas de mortalidad que limitaron su beneficio en los pacientes mayores de 40 años $^{17}$. Con el advenimiento de protocolos no mieloablativos basados en fludarabina es posible ofrecer este tipo de terapia a los pacientes de mayor edad, obteniendo tasas de curación muy similares a las de los pacientes menores ${ }^{12}$; como consecuencia, este límite es motivo de discusión.

La experiencia aquí descrita reproduce en nuestro medio los resultados obtenidos en otros países. La SG expuesta aquí es muy similar a la de otros grupos internacionales ${ }^{6-10,12-13,18}$, lo cual representa un resultado excelente si se tiene en cuenta que el $43 \%$ de nuestros pacientes se encontraban politransfundidos; además, el tiempo promedio entre el diagnóstico de SFM y el TAPH resultó muy superior a lo reportado en la literatura y en el $47 \%$ de los casos fue superior a un año. A pesar de que en el presente análisis ni el estado de politransfusión ni el tiempo SFM-TAPH resultaron en un impacto significativo sobre la SG, múltiples estudios han demostrado que estos tienen una repercusión negativa sobre la supervivencia2, $2,6-9,19$.

La fuente ideal de progenitores hematopoyéticos para un TAPH en los SFM sigue siendo controversial. En un esfuerzo conjunto, el registro del Center for International Blood and Marrow Transplant Research de los Estados Unidos (CIBMTR) y el registro del European Group for Blood and Marrow Transplantation (EBMT) revisaron retrospectivamente los desenlaces de 692 trasplantes alogénicos en pacientes con ASMO, encontrando que la utilización de sangre periférica como fuente de $\mathrm{PH}$ aumentaba significativamente la incidencia acumulada de EICHa grados II a IV y EICHc en comparación con los PH obtenidos de la médula ósea con y sin estimulación con factores de crecimiento granulocítico (EICHa: 28\% frente al $14 \%$ y $13 \%$; EICHc: $43 \%$ frente al $10 \%$ y $16 \%$, respectivamente).

No obstante, la supervivencia solo fue superior al utilizar PH obtenidos de médula ósea en los pacientes menores de 21 años. No hubo ningún beneficio en la velocidad del injerto ni en la mortalidad relacionada con el procedimiento que se empleó de PH de sangre periférica ${ }^{20}$. Pese a estos resultados, es de resaltar las dificultades técnicas y riesgos asociados que implican para un donante sano la obtención de PH de su médula ósea, por lo cual muchos centros de referencia en TAPH, incluyendo la UTMO de Marly, continúan utilizando hoy en día la sangre periférica como fuente de $\mathrm{PH}$. Igualmente, algunas experiencias sugieren que la adición de ATG a los regímenes de acondicionamiento podría disminuir el impacto de la $\mathrm{EICH}$ al usar los $\mathrm{PH}$ provenientes de sangre periférica ${ }^{21}$.

El número de trasplantes realizados con médula ósea en esta cohorte representó únicamente el 8,1\%, por lo cual fue imposible hacer un análisis del impacto de la fuente sobre la variable principal. La baja mortalidad relacionada con la EICHa en los pacientes acondicionados con los regímenes que generan depleción linfoide (CyATG: 13\%, Cy-Flu-ATG: 5\%, alemtuzumab: 0\%) y con fludarabina sugieren que este tipo de medicamentos podrían contrarrestar el efecto negativo al usar PH de sangre periférica en la frecuencia de $\mathrm{EICH}$.

Los reportes de los grupos de experiencia en TAPH en India (que implican un contexto socioeconómico similar al nacional) enfrentan limitaciones homólogas, registrando demoras importantes en el tiempo entre el diagnóstico y el TAPH y el uso de la sangre periférica como fuente de $\mathrm{PH}$ en cerca del $80 \%$ de los pacientes. El uso del protocolo Cy-Flu-ATG se ha visto asociado a una tasa de EICHa del 29\%, de la cual solo el $6 \%$ es grado III-IV22.

La ATG se adicionó inicialmente al acondicionamiento con la finalidad de disminuir las tasas de falla del injerto. En los primeros reportes fue evidente una mejoría en la supervivencia a cinco años en comparación con los resultados históricos (92\% vs. $72 \%$ ). Posteriormente, el seguimiento a largo plazo confirmó los hallazgos (supervivencia del $88 \%$ a seis años) ${ }^{11,23}$. Actualmente, la mayoría de los autores coinciden en el beneficio obtenido con el uso ATG, sin embargo, algunos estudios aleatorizados sugieren que esta sólo es útil como parte de los regímenes de acondicionamiento en los TAPH obtenidos de sangre periférica ${ }^{24,25}$. En nuestra experiencia, el empleo de ATG dentro del acondicionamiento tuvo un impacto importante, evidente en una mejor SG y en una menor tasa de EICHa.

La adición de fludarabina a los acondicionamientos para SFM se concibió para obtener un mayor efecto 
inmunosupresor y disminuir la toxicidad tisular asociada con el uso de irradiación corporal total (TBI, por su sigla en inglés) o las altas dosis de Cy ${ }^{26}$, en un intento por mejorar los resultados en pacientes sometidos a trasplante con $\mathrm{PH}$ de donantes no familiares y para disminuir el riesgo de falla del injerto en pacientes politransfundidos ${ }^{27,28}$. A esta combinación se le adicionó además ATG y se redujo la dosis de Cy, lo que resultó en tasas de falla del injerto menores al $3 \%$, junto a una baja mortalidad relacionada con el procedimiento 22 . Estos resultados estimularon a otros grupos a utilizar la combinación Cy-Flu-ATG en otras poblaciones de alto riesgo, tales como los niños con anemia de Fanconi, en quienes se ha demostrado que la adición de fludarabina al protocolo de condicionamiento mejora significativamente la supervivencia ( $86 \%$ vs. 59\%, $\mathrm{p}<0.05)^{29}$.

En nuestro servicio, en el año 2006, dos pacientes presentaron falla secundaria del injerto; uno de ellos recibió acondicionamiento con Cy-ATG y el otro con un protocolo basado en alemtuzumab. Ambos suspendieron voluntariamente la inmunosupresión tempranamente después del trasplante. Para el segundo trasplante, teniendo en cuenta el alto riesgo de falla del injerto, se decidió utilizar como acondicionamiento Cy-Flu-ATG, soportado por la experiencia reportada por otros centros. Los resultados en estos dos casos (considerados de alto riesgo) fueron muy alentadores en términos de la tolerancia y de la documentación del injerto tempranamente.

A partir de ese momento, se adoptó la combinación Cy-Flu-ATG como el protocolo estándar de acondicionamiento para todos los pacientes con SFM tratados en la UTMO. La justificación de esta decisión fue soportada por las condiciones de nuestro sistema de salud, en donde la mayoría de casos con indicación de TPH para
SFM tienen basalmente más de un factor de riesgo para falla del injerto. Como ha sido reportado por otros grupos, el uso de la combinación Cy-Flu-ATG tanto en pacientes pediátricos como adultos ha resultado en un menor tiempo de hospitalización, menor requerimiento transfusional, de antibioticoterapia y de días de alimentación parenteral. La tasa de falla del injerto fue baja y la mortalidad relacionada con el procedimiento ha sido menor al 5\% $22,30,31$.

Este informe incluye a 15 pacientes mayores de 40 años, con una supervivencia del $73.3 \%$, lo cual resulta consistente con otras experiencias de TAPH para esta población. Es relevante recalcar que más de la mitad de ellos recibió como acondicionamiento la combinación Cy-Flu-ATG, ya que este protocolo nos permitió trasplantar pacientes severamente enfermos de altísimo riesgo no solo por su edad, sino por múltiples comorbilidades, con una mortalidad muy baja. Otros grupos han reportado cómo el uso de protocolos no mieloablativos basados en fludarabina mejoran significativamente la supervivencia en mayores de 40 años (77\% vs. 60\%, $p=0.14)$ y disminuyen el riesgo de falla en el injerto $(0 \% \text { vs. } 11 \%)^{12}$.

\section{Conclusión}

El TAPH en nuestro medio se constituye en una alternativa real de curación para este grupo de pacientes, con una supervivencia cercana al $70 \%$, e incluso superior al $90 \%$, con el uso de protocolos no mieloablativos basados en fludarabina y con las condiciones de soporte actuales, que, además, se asocian con menor frecuencia de EICHa. En pacientes mayores de 40 años, de alto riesgo, los resultados son similares, lo que permite considerar, hoy en día, el trasplante como terapia de primera línea en este grupo si se dispone de un donante HLA idéntico intrafamiliar.

\section{Referencias}

1. Young NS, Maciejewski J. The pathophysiology of acquired aplastic anemia. N Engl J Med. 1997;336(19):1365-72.

2. Marsh JC, Ball SE, Cavenagh J, Darbyshire P, Dokal I, GordonSmith EC, et al. Guidelines for the diagnosis and management of aplastic anaemia. Br J Haematol. 2009;147(1):43-70.

3. Sanders JE, Storb R, Anasetti C, Deeg HJ, Doney K, Sullivan $\mathrm{KM}$, et al. Marrow transplant experience for children with severe aplastic anemia. Am J Pediatr Hematol Oncol. 1994;16(1):43-9.
4. Medeiros LA, Pasquini R. Anemia aplásica adquirida e anemia de Fanconi: diretrizes brasileiras em transplante de células-tronco hematopoéticas. Rev Bras Hematol Hemoter. 2010;32(supl 1):40-5.

5. Deeg HJ, Leisenring W, Storb R, Nims J, Flowers ME, Witherspoon RP, et al. Long-term outcome after marrow transplantation for severe aplastic anemia. Blood. 1998;91(10):3637-45.

6. Gluckman E, Socie G, Devergie A, Bourdeau-Esperou H, Traineau $R$, Cosset JM. Bone marrow transplantation in 107 pa- 
tients with severe aplastic anemia using cyclophosphamide and thoraco-abdominal irradiation for conditioning: long-term follow-up. Blood. 1991;78(9):2451-5.

7. Bacigalupo A, Brand R, Oneto R, Bruno B, Socie G, Passweg J, et al. Treatment of acquired severe aplastic anemia: bone marrow transplantation compared with immunosuppressive therapy--The European Group for Blood and Marrow Transplantation experience. Semin Hematol. 2000;37(1):69-80.

8. Arranz R, Conde E, Rodriguez-Salvanes F, Pajuelo FJ, Cabrera R, Sanz MA, et al. CsA-based post-graft immunosuppression: the main factor for improving outcome of allografted patients with acquired aplastic anemia. A retrospective survey by the Spanish Group of Hematopoietic Transplantation. Bone Marrow Transplant. 2002;29(3):205-11.

9. Ades L, Mary JY, Robin M, Ferry C, Porcher R, Esperou $H$, et al. Long-term outcome after bone marrow transplantation for severe aplastic anemia. Blood. 2004;103(7):2490-7.

10. Kim I, Yoon SS, Park S, Kim BK, Kim NK. The treatment of severe aplastic anemia: outcomes of bone marrow transplantation and immunosuppressive therapy in a single institution of Korea. J Korean Med Sci. 2003;18(3):365-71.

11. Storb R, Blume KG, O’Donnell MR, Chauncey T, Forman SJ, Deeg $\mathrm{HJ}$, et al. Cyclophosphamide and antithymocyte globulin to condition patients with aplastic anemia for allogeneic marrow transplantations: the experience in four centers. Biol Blood Marrow Transplant. 2001;7(1):39-44.

12. Sangiolo D, Storb R, Deeg HJ, Flowers MED, Martin PJ, Sandmaier BM, et al. Outcome of allogeneic hematopoietic cell transplantation from HLA-identical siblings for severe aplastic anemia in patients over 40 years of age. Biol Blood Marrow Transplant. 2010;16(10):1411-8

13. Maury S, Bacigalupo A, Anderlini P, Aljurf M, Marsh J, Socie G, et al. Improved outcome of patients older than 30 years receiving HLA-identical sibling hematopoietic stem cell transplantation for severe acquired aplastic anemia using fludarabine-based conditioning: a comparison with conventional conditioning regimen. Haematologica. 2009;94(9):1312-5.

14. Glucksberg H, Storb R, Fefer A, Buckner CD, Neiman PE, Clift $R A$, et al. Clinical manifestations of graft-versus-host disease in human recipients of marrow from HL-A-matched sibling donors. Transplantation. 1974;18(4):295-304.

15. Champlin RE, Horowitz MM, van Bekkum DW, Camitta BM, Elfenbein GE, Gale RP, et al. Graft failure following bone marrow transplantation for severe aplastic anemia: risk factors and treatment results. Blood. 1989;73(2):606-13.

16. Camitta BM, Storb R, Thomas ED. Aplastic anemia (first of two parts): pathogenesis, diagnosis, treatment, and prognosis. $\mathrm{N}$ Engl J Med. 1982;306(11):645-52.

17. Locasciulli A, Oneto R, Bacigalupo A, Socié G, Korthof E, Bekassy $A$, et al. Outcome of patients with acquired aplastic anemia given first line bone marrow transplantation or immunosuppressive treatment in the last decade: a report from the European Group for Blood and Marrow Transplantation. Haematologica. 2007;92(1):11-8.

18. Barriga $C F$, Wietstruck $P A$, Becker $K A$, Zúñiga $C P$, de Besa $C P$, Álvarez ZM, et al. Tratamiento de anemia aplásica severa adquirida en pacientes pediátricos con inmunosupresión y trasplante alogénico de precursores hematopoyéticos. [Treatment of acquired severe aplastic anemia in pediatric patients with immunosupression and allogeneic stem cell hematopoyetic transplant]. Rev Med Chil. 2007;135(11):1421-8.
19. Passweg JR, Socie G, Hinterberger W, Bacigalupo A, Biggs JC, Camitta BM, et al. Bone marrow transplantation for severe aplastic anemia: has outcome improved? Blood. 1997;90(2):858-64.

20. Chu R, Brazauskas R, Kan F, Bashey A, Bredeson C, Camitta $B$, et al. Comparison of outcomes after transplantation of GCSF-stimulated bone marrow grafts versus bone marrow or peripheral blood grafts from HLA-matched sibling donors for patients with severe aplastic anemia. Biol Blood Marrow Transplant. 2011;17(7):1018-24.

21. Ghavamzadeh A, Alimoghaddam K, Hamidieh AA, Jalili $M$, Bahar B, Iravani M, et al. Peripheral blood stem cell transplantation versus bone marrow transplantation in severe aplastic anemia. Biol Blood Marrow Transplant. 2011;17(2):S291.

22. George B, Mathews V, Viswabandya A, Kavitha ML, Srivastava $A$, Chandy $M$. Fludarabine and cyclophosphamide based reduced intensity conditioning (RIC) regimens reduce rejection and improve outcome in Indian patients undergoing allogeneic stem cell transplantation for severe aplastic anemia. Bone Marrow Transplant. 2007;40(1):13-8.

23. Storb R, Etzioni R, Anasetti C, Appelbaum F, Buckner CD, Bensinger $W$, et al. Cyclophosphamide combined with antithymocyte globulin in preparation for allogeneic marrow transplants in patients with aplastic anemia. Blood. 1994;84(3):941-9.

24. Champlin RE, Perez WS, Passweg JR, Klein JP, Camitta BM, Gluckman E, et al. Bone marrow transplantation for severe aplastic anemia: a randomized controlled study of conditioning regimens. Blood. 2007;109(10):4582-5.

25. Champlin RE, Perez WS, Passweg J, Klein JP, Camitta BM, Gluckman E, et al. Addition of antithymocyte globulin (ATG) to cyclophosphamide (Cy) for HLA-identical sibling allogeneic bone marrow transplantation (BMT) for severe aplastic anemia (SAA): results of a randomized controlled trial. Blood. 2003;102(11):80a.

26. Kapelushnik J, Or R, Slavin S, Nagler A. A fludarabine-based protocol for bone marrow transplantation in Fanconi's anemia. Bone Marrow Transplant. 1997;20(12):1109-10.

27. Bacigalupo A, Locatelli F, Lanino E, Marsh J, Socie G, Maury S, et al. Fludarabine, cyclophosphamide and anti-thymocyte globulin for alternative donor transplants in acquired severe aplastic anemia: a report from the EBMT-SAA Working Party. Bone Marrow Transplant. 2005;36(11):947-50.

28. Kumar R, Prem S, Mahapatra M, Seth T, Chowdhary DR, Mishra $P$, et al. Fludarabine, cyclophosphamide and horse antithymocyte globulin conditioning regimen for allogeneic peripheral blood stem cell transplantation performed in non-HEPA filter rooms for multiply transfused patients with severe aplastic anemia. Bone Marrow Transplant. 2006;37(8):745-9.

29. Locatelli F, Zecca M, Pession A, Morreale G, Longoni D, Di Bartolomeo P, et al. The outcome of children with Fanconi anemia given hematopoietic stem cell transplantation and the influence of fludarabine in the conditioning regimen: a report from the Italian pediatric group. Haematologica. 2007;92(10):1381-8.

30. Ringden O, Remberger M, Svenberg P, Svahn BM, Dahllof G, Gustafsson B, et al. Fludarabine-based disease-specific conditioning or conventional myeloablative conditioning in hematopoietic stem cell transplantation for treatment of non-malignant diseases. Bone Marrow Transplant. 2007;39(7):383-8.

31. George B, Mathews V, Viswabandya A, Kavitha ML, Srivastava $A$, Chandy M. Fludarabine based reduced intensity conditioning regimens in children undergoing allogeneic stem cell transplantation for severe aplastic anemia. Pediatr Transplant. 2008;12(1):14-9. 